\title{
Efectos del Síndrome de Apneas- Hipopneas del Sueño sobre la calidad de vida y la somnolencia diurna*
}

\author{
Obstructive Sleep Apnea Syndrome Effects on \\ Quality of Life and Daytime Sleepiness
}

Recibido: noviembre 24 de 2011 | Revisado: julio 9 de 2012 | Aceptado: diciembre 5 de 2012

\author{
OtTAVIA GUGLIELMI ** \\ ANA ISABEL SÁNCHEZ GÓMEZ *** \\ Universidad de Granada, España \\ BERNABÉ JURADO-GÁMEZ \\ GUALBERTO BUEla-CASAL
}

Hospital Universitarios Reina Sofia de Córdoba, España

doi:10.11144/Javeriana.UPSY12-2.esah

Para citar este artículo: Guglielmi, O., Sánchez, A. I., Jurado-Gámez, B. \& Buela-Casal, G. (2013). Efectos del Síndrome de Apneas-Hipopneas del Sueño sobre la calidad de vida y la somnolencia diurna. Universitas Psychologica, 12(2), 601-611.

* Artículo de investigación.

** Facultad de Psicología. Universidad de Granada, España. Campus de Cartuja, s/n E-18071 Granada. ottavia@ugr.es

**** Universidad de Granada, España. Profesora Titular. Departamento de Personalidad, Evaluación y Tratamiento Psicológico. E-mail: aisabel@ugr.es

\section{RESUMEN}

El objetivo de esta investigación fue evaluar en pacientes con Síndrome de Apneas-Hipopneas del Sueño (SAHS), la calidad de vida y el impacto de la somnolencia diurna. Se incluyeron 100 sujetos (50 pacientes con SAHS y 50 sujetos sanos) que respondieron al Cuestionario de Salud (SF-36), Cuestionario de Impacto Funcional del Sueño (FOSQ) y a la Escala de Somnolencia de Epworth (ESE). Respecto al grupo control, el grupo clínico mostró diferencias estadísticamente significativas $(p<0.05)$ en la mayoría de las variables estudiadas. Los factores más influyentes fueron el índice de masa corporal, el índice de apneas-hipopneas y el índice de desaturación. Este estudio demuestra la importante repercusión que el SAHS tiene en la calidad de vida de los pacientes.

Palabras clave autor

Síndrome de Apneas-Hipopneas del Sueño, calidad de vida, polisomnografía, excesiva somnolencia diurna.

Palabras clave descriptores

Calidad de sueño, psicología de la salud, investigación cuantitativa.

\section{A B S T R A C T}

The objective of this research was to evaluate quality of life and impact of daytime sleepiness in patients with Sleep Apnea/Hypopnea Syndrome (SAHS). 100 subjects were included: 50 patients with OSAS and 50 healthy subjects, who responded to the Short Form-36 Health Survey (SF-36), the Functional Outcomes of Sleep Questionnaire (FOSQ) and the Epworth Sleepiness Scale (ESS). The comparison between clinical and control group showed statistically significant differences $(p<0.05)$ in most of the variables studied. The results found that body mass index, apnea-hypopnea index and desaturation index were the most important affecting factors. This study shows the great impact OSAS has on the quality of life of patients. Key words authors

Sleep Apnea-Hipopnea Syndrome, Quality of Life, Excessive Daytime Somnolence, Polysomnography.

Key words plus

Sleep Quality, Health Psychology, Quantitative Psychology. 


\section{Introducción}

El Síndrome de Apneas-Hipopneas del Sueño (SAHS) se caracteriza por episodios recurrentes durante el sueño de limitación al flujo aéreo en la vía aérea superior que provocan descensos en la saturación de oxígeno $\left(\mathrm{SaO}_{2}\right)$ y microdespertares (Grupo Español de Sueño, 2005).

EL SAHS es más frecuente en hombres que en mujeres, con una relación hombre- mujer de 7-20:1. En el contexto de países desarrollados, es más común en adultos entre los 40 y 50 años, aunque también se observa en niños, adolescentes y personas de la tercera edad (Páez \& Londoño, 2011). Estudios epidemiológicos demuestran una importante prevalencia del SAHS en la población general adulta de mediana edad. Así pues, se estima que la prevalencia del síndrome, definido por un índice de apneas-hipopneas por hora de sueño $\geq$ 5 , asociado a excesiva somnolencia diurna, se sitúa en la población general entre el 3-7 \% en hombres y el 2-5\% en mujeres (Lurie, 2011). Hay evidencias que demuestran la relación entre el SAHS y el desarrollo de hipertensión, trastornos cardiovasculares, cerebrovasculares y cambios en el metabolismo (McArdle, Hillman, Beilin \& Watts, 2007).

La sintomatología del SAHS es muy heterogénea e incluye entre otros síntomas, ronquidos, excesiva somnolencia diurna, déficit cognitivos, cambios en el estado de ánimo, problemas sexuales, fragmentación de sueño, despertares nocturnos, así como cambios en la arquitectura del sueño (Guglielmi, Sánchez, Jurado-Gámez, Buela-Casal \& Bardwell, 2011; McArdle \& Douglas, 2001; Páez $\&$ Londoño, 2011). En un estudio reciente se ha demostrado que los principales predictores clínicos y de SAHS son la edad mayor de 45 años, el sexo masculino, un índice de masa corporal mayor de $26 \mathrm{~kg} / \mathrm{m}^{2}$, además de una historia de tabaquismo, una duración de los síntomas de más de dos años, informe de apneas por testigos y excesiva somnolencia diurna (Saldías, Jorquera \& Díaz, 2010).

Algunos de los síntomas del SAHS pueden afectar la calidad de vida de los enfermos. Diferentes trabajos han relacionado el sueño con la percepción general de la salud y de la calidad de vida (Marín-
Agudelo, Franco, Vinaccia-Alpi, Tobón \& Sandín, 2008; Miró, Cano-Lozano \& Buela-Casal, 2005). En la actualidad no existen conclusiones claras con respecto a la relación entre calidad de vida y gravedad del SAHS, aunque se postula que la causa puede ser multifactorial (Sforza, Janssens, Rochat \& Ibáñez, 2003), y que el deterioro en la calidad de vida parece ser proporcional a la severidad del síndrome (Lópes, Esteves, Bittencourt, Tufik \& Mello, 2008).

Como se ha comentado anteriormente, las manifestaciones clínicas del SAHS son muy variadas. La excesiva somnolencia diurna es el síntoma que mayor repercusión tiene en la calidad de vida de los pacientes, afectando tanto a la capacidad laboral del paciente, como a su capacidad de conducción de vehículos a motor (Sánchez, Martínez, Miró, Bardwell $\&$ Buela-Casal, 2009), lo que ha provocado que en los últimos años diversos estudios hayan intentado identificar qué factores son responsables del incremento en los accidentes de tráfico asociados a esta patología de sueño (Sánchez, Miró \& Martínez, 2006). La fragmentación del sueño y la hipoxemia nocturna apuntan como las principales causas de la somnolencia diurna (Mediano et al., 2007), si bien su relación con los índices de calidad de sueño y con variables respiratorios es controvertida.

La hipótesis subyacente en este trabajo es que los pacientes con trastornos respiratorios durante el sueño pueden mostrar mayor somnolencia diurna, peor calidad de vida y mayor repercusión sobre el funcionamiento diario respecto al grupo control. Para evaluar esta hipótesis, se diseña un estudio con el objetivo de analizar qué variables asociadas a la gravedad del SASH se relacionan con el deterioro de la calidad de vida y la somnolencia diurna, así como evaluar la repercusión de esta última en las actividades diarias de los pacientes. Para la redacción de este manuscrito se ha seguido los criterios propuestos por Ramos-Álvarez, Moreno-Fernández, Valdés-Conroy y Catena (2008).

\section{Método}

Se realiza un estudio tipo ex post facto prospectivo (Montero \& León, 2007), que compara los resul- 
tados de pacientes con SAHS respecto a otros sujetos sin esta enfermedad que se incluyen en el grupo control.

\section{Participantes}

La muestra estuvo formada por 100 sujetos (50 pacientes con SAHS y 50 sujetos de control). La distribución por sexo en los dos grupos fue idéntica: 35 hombres y 15 mujeres, con edades comprendidas entre los 35 y los 65 años $(M=52.91$ años; $D E=8.38)$. El índice de masa corporal medio (IMC) fue de $30.4 \pm 6.4$. No se encontraron diferencias estadísticamente significativas en la edad media de los diferentes grupos $(t=0.75$; $p>0.05)$, aunque ambos grupos sí difirieron en el $\operatorname{IMC}(t=-4,30 ; p<0.01)$.

Los pacientes del grupo clínico se seleccionaron de manera consecutiva de la población remitida a la Unidad del Sueño de un Hospital Universitario por presentar sospecha clínica de SAHS. El diagnóstico de SAHS se efectuó tras realizar una polisomnografía diagnóstica de noche completa. El grupo control fue seleccionado de los acompañantes de los enfermos que acudían a la consulta externa del mismo hospital, a los que se les descartó la existencia de un SAHS con un cuestionario clínico y una pulsioximetría nocturna de registro continuo (Pulsox 300i Konika). A los sujetos se les realizó una serie de preguntas para valorar la existencia de SAHS, no debiendo presentar ninguno de lo siguientes síntomas: ronquido, paradas respiratorias observadas durante el sueño, despertar por ahogo, sueño no reparador o tendencia al sueño en situaciones inapropiadas. Además, a los sujetos que no presentaban ninguno de estos síntomas se les realizó una pulsioximetría nocturna y se excluyeron aquellos que mostraron un número de descensos en la saturación de oxigeno $>$ $3 \%$ (ID3) por encima de 5/hora, considerando que estos sujetos pudieran tener algún tipo de trastorno respiratorio durante el sueño. En ambos grupos, se excluyeron del estudio a personas con enfermedad mental limitante como psicosis o demencia, enfermedades neurológicas, insuficiencia respiratoria crónica, insuficiencia cardiaca, enfermedad renal crónica y trastornos del sueño secundarios a otras patologías que puedan afectar la calidad del sueño. Se excluyeron también sujetos con adicción a alcohol y drogas o en tratamiento con fármacos neurolépticos, tranquilizantes u otros medicamentos que puedan alterar el sueño.

\section{Instrumentos}

El estudio se realizó con un polisomnógrafo Somnoscreen (Somnomedic, Ltd. Germany) y se utilizaron dos canales de electroencefalograma (C4-A1 y C3-A2), dos canales de electrooculograma, dos canales de electromiografía, un canal de electrocardiograma. Se analizaron también la saturación del oxígeno, el pulso, la frecuencia de los ronquidos, el flujo aéreo por medio de cánula de presión y termistor, el esfuerzo toráxico y abdominal y los cambios en la posición del cuerpo. Las señales electrofisiológicas y de polisomnografía se analizaron por medio del programa DOMINO.

Para evaluar la calidad de vida se utilizó la versión adaptada al español por Alonso, Prieto y Antó (1995) del Cuestionario de Salud SF-36 (SF-36 Health Survey) de Ware y Sherbourne (1992). El SF-36 es un cuestionario autoadministrado de 36 ítems que valora la salud a través de ocho dimensiones que se agrupan en dos valores resumen: sumario físico y sumario psíquico. Las ocho dimensiones del estado de salud son: Función Física, Rol Físico, Dolor, Salud General, Vitalidad, Funcionamiento Social, Rol Emocional y Salud Mental. Posee una elevada consistencia interna con alfa de Cronbach superior a 0.75 en todas las escalas, excepto en la subescala de funcionamiento social y la validez testretest es de 0.8 para la función física, la vitalidad y la percepción general de salud y de 0.6 para la función social (Alonso et al., 1998).

Mediante la versión española de Ferrer et al. (1999) del Cuestionario del Impacto Funcional del Sueño (Functional Outcomes of Sleep Questionnaire [FOSQ]) de Weaver et al. (1997), se evaluó la repercusión de la somnolencia diurna en las actividades de la vida diaria. El FOSQ consta de 30 ítems divididos en cinco subescalas: Productividad General, Productividad Social, Nivel de Actividad, Vigilancia, Intimidad y Relaciones Sexuales. Las 
opciones de respuesta varían entre 0 (no aplicable) a 4 (ninguna dificultad). La consistencia interna superó el estándar de 0.9 (Ferrer et al., 1999).

Finalmente, la excesiva somnolencia diurna se midió con la versión española de Chiner, Arriero, Signes-Costa, Marco y Fuentes (1999) de la Escala de Somnolencia de Epworth (Epworth Sleepiness Scale [ESS]) de Johns (1991). La ESS es un cuestionario que evalúa la tendencia de una persona a quedarse dormida en ocho situaciones diferentes (viendo la televisión, leyendo, conduciendo). Cada uno de los ítems tiene varias opciones de respuesta, con puntuaciones que oscilan entre $(0=$ nunca, 1 = leve, 2 = moderado y $3=$ severo). Puntajes altos representan mayor grado de somnolencia. Estudios psicométricos indican que la ESS tiene un coeficiente de correlación de Cronbach superior 0.9 (Ferrer et al., 1999).

\section{Procedimiento}

A cada paciente perteneciente al grupo clínico se le realizó una pequeña entrevista y se consultó su historia clínica, lo que permitió recabar algunos datos sobre su edad, IMC, profesión, consumo de tabaco, alcohol, etc. Los cuestionarios fueron administrados por los técnicos de la Unidad del Sueño antes de que los sujetos se acostasen. Todos los sujetos fueron informados del estudio a través de una hoja informativa. Para la suministración del cuestionario a los sujetos del grupo control, se adoptaron modalidades similares y fueron entrevistados por el investigador.

El diagnostico de SASH se realizó mediante polisomnografia convencional de noche completa, durante el periodo comprendido entre abril y septiembre de 2008. Los estudios de sueño fueron corregidos manualmente según las recomendaciones de Rechtschaffen y Kales (1968). En concordancia con el consenso nacional sobre el SAHS (Grupo Español de Sueño, 2005), se consideró apnea el cese de la señal respiratoria mayor del $90 \%$ de al menos 10 segundos de duración, e hipopnea una reducción de la amplitud de la señal respiratoria entre el $30 \%$ y el $90 \%$, una duración mayor a 10 segundos y acompañada por una desaturación mayor al $3 \%$ y/o un microdespertar en el electroencefalograma. El diagnostico de SAHS fue determinado por el índice de apneas-hipopneas (IAH), considerándose que en un paciente con síntomas compatibles y un IAH $\geq$ 5 es diagnóstico de SAHS (Grupo Español de Sueño, 2005). El proyecto de investigación fue aprobado por el comité ético del Hospital Universitario Reina Sofía de Córdoba (España) y solamente las personas que firmaron el consentimiento informado participaron al estudio. El procedimiento del estudio se desarrolló en todo momento en concordancia con la Declaración de Helsinki.

\section{Análisis de datos}

Los análisis estadísticos se realizaron con el programa SPSS 15.0 (SPSS Inc., Chicago, IL, EE. UU.). En todos los análisis se utilizaron niveles de significación inferiores o iguales a 0.05. Se calcularon los estadísticos descriptivos básicos (media, desviación típica, puntuación máxima y mínima) de las variables contempladas en el estudio. Con el fin de explorar las diferencias entre los grupos (pacientes con apnea y controles sanos) en las diversas variables clínicas (calidad de vida, impacto funcional del sueño y somnolencia), se recurrió a la prueba $t$ de Student para muestras independientes. Para examinar la relación entre el IMC y las variables polisomnográficas con el resto de medidas clínicas (impacto funcional del sueño, somnolencia y calidad de vida) en el grupo de pacientes con SAHS, se recurrió al coeficiente de correlación de Pearson para muestras independientes. Finalmente, se efectuó una regresión lineal múltiple para encontrar las variables que mejor predicen la somnolencia y el funcionamiento diario de los pacientes con SASH.

\section{Resultados}

En la Tabla 1 se presenta un resumen de las variables respiratorias y de calidad de sueño en el grupo de pacientes con SAHS. El $18 \%$ de los sujetos presentó un SAHS leve; el $18 \%$, moderado; el $14 \%$, grave y, finalmente, en el $50 \%$ de los casos el SAHS era muy grave. 
TABLA 1

Medias y desviaciones típicas de las variables respiratorias y de calidad de sueño en el grupo de pacientes con SAHS

\begin{tabular}{|c|c|c|}
\hline Variables & Rango de puntuación & $M(D E)$ \\
\hline $\mathrm{IAH}$ & $6.40-119.30$ & $46.96(31.2)$ \\
\hline $\mathrm{SaO}_{2}$ basal & $(90-97)$ & $93.84(1.44)$ \\
\hline ID $3^{2}$ & $(6.40-128.00)$ & $47.53(32.8)$ \\
\hline TST & $(58-380)$ & $288.42(51.89)$ \\
\hline Eficiencia & $(30-97)$ & $81.41(12.79)$ \\
\hline \% Sueño REM & $(00-27.80)$ & $10.27(7.5)$ \\
\hline \% Sueño No REM & $(72.20-100)$ & $89.74(7.61)$ \\
\hline Índice de arousal & $(5.70-93.90)$ & $27.15(17.63)$ \\
\hline
\end{tabular}

Notas. IAH: Índice apneas e hipopneas por hora de sueño; ID3: número de desaturaciones > 3 \%; TST: tiempo total de sueño; \% Sueño REM: sueño REM/TST \%; \% Sueño no REM: sueño no REM/TST \%.

Fuente: elaboración propia.

Análisis comparativo de los índices

de impacto funcional del sueño,

calidad de vida y somnolencia

Un resumen de los estadísticos descriptivos de las distintas variables del estudio para ambos grupos (apnea y control) se recoge en la Tabla 2. Respecto a las escalas de calidad de vida del SF-36, los grupos clínicos y control difirieron de manera significativa en todas ellas (valores de $t$ entre $-4.65, p<0.01$ y $-2.19, p<0.05)$, a excepción del dolor físico $(p>0.05)$. En cuanto a las medidas del FOSQ, los dos grupos también difieren de forma significativa (valores de $t$ entre $-7.01 p<0.01$ y $-3.86, p<0.01$ ) en todas las dimensiones medidas. Por último, y en relación a la somnolencia diurna, se observan igual-

TABLA 2

Comparación entre los grupos en calidad de vida, impacto funcional del sueño y somnolencia

\begin{tabular}{|c|c|c|c|}
\hline Variables & $\begin{array}{c}\text { SAHS N }=50 \\
M(D E)\end{array}$ & $\begin{array}{c}\text { Control } N=50 \\
M(D E)\end{array}$ & $t$ \\
\hline \multicolumn{4}{|l|}{ SF-36 } \\
\hline Función física & $70.06(29.78)$ & $91.6(13.53)$ & $-4.65 * *$ \\
\hline Rol físico & $63.37(35.64)$ & $85(22.30)$ & $-3.63 * *$ \\
\hline Dolor & $65.46(30.52)$ & $72.56(29.24)$ & -1.18 \\
\hline Salud general & $48.79(21.72)$ & $65.02(15.66)$ & $-4.19 * *$ \\
\hline Vitalidad & $50.29(25.5)$ & $64.92(21.07)$ & $-3.10 * *$ \\
\hline Función social & $68(28.25)$ & $79(21.49)$ & $-2.19 *$ \\
\hline Rol emocional & $69.83(31.49)$ & $84.83(22.63)$ & $-2.73 * *$ \\
\hline Salud mental & $62.47(21.02)$ & $75.61(17.6)$ & $-3.36 * *$ \\
\hline Sumario de salud física & $45.14(10.56)$ & $51.49(8.47)$ & $-3.29 *$ \\
\hline Sumario de salud mental & $43.33(13.49)$ & $48.82(10.05)$ & $-2.92 *$ \\
\hline \multicolumn{4}{|l|}{ FOSQ } \\
\hline Productividad general & $15.93(4.67)$ & $21.41(3.23)$ & $-6.81 * *$ \\
\hline Productividad social & $19.36(5.79)$ & $22.98(3.19)$ & $-3.86 * *$ \\
\hline Nivel de actividad & $14.19(6.15)$ & $20.52(4.34)$ & $-5.94 * *$ \\
\hline Vigilancia & $15.05(6.57)$ & $21.75(4.16)$ & $-6.08 * *$ \\
\hline Intimidad & $14.82(8.17)$ & $21.75(5.22)$ & $-5.01 * *$ \\
\hline FOSQ total & $77.54(26.4)$ & $107.17(14.01)$ & $-7.01 * *$ \\
\hline ESE & $13(5.27)$ & $5.8(2.97)$ & $8.30 * *$ \\
\hline
\end{tabular}

Nota. $* p<0.05 ; * *<0.01$; Cuestionario de Salud SF-36; Cuestionario del Impacto Funcional del Sueño FOSQ; ESE: Escala de somnolencia de Epworth.

Fuente: elaboración propia. 
TABLA 3

Correlaciones entre las puntuaciones del FOQS y la ESE con las variables polisomnográficas, respiratorias y el IMC en el grupo de pacientes con SAHS

\begin{tabular}{lccccccc}
\hline & \multicolumn{9}{c}{ Cuestionario de Impacto Funcional de Sueño (FOSQ) } & \multicolumn{2}{c}{ ESE } \\
\hline & $\begin{array}{c}\text { Productividad } \\
\text { general }\end{array}$ & $\begin{array}{c}\text { Productividad } \\
\text { Social }\end{array}$ & $\begin{array}{c}\text { Nivel de } \\
\text { actividad }\end{array}$ & Vigilancia & Intimidad & Total & \\
\hline IAH & -0.22 & -0.23 & -0.23 & -0.22 & -0.19 & -0.27 & $0.29^{*}$ \\
$\mathrm{SaO}_{2}$ basal & 0.11 & -0.05 & 0.14 & 0.10 & 0.21 & 0.13 & -0.13 \\
$\mathrm{ID} 3$ & $-0.30^{*}$ & -0.27 & $-0.29^{*}$ & -0.26 & -0.24 & $-0.33^{*}$ & $-0.35^{*}$ \\
$\mathrm{TST}$ & 0.14 & 0.04 & 0.18 & -0.02 & 0.17 & 0.14 & -0.08 \\
Eficiencia & 0.12 & 0.05 & 0.16 & -0.08 & 0.25 & 0.14 & -0.03 \\
$\%$ REM & 0.26 & 0.10 & 0.23 & -0.01 & $0.44^{* *}$ & 0.22 & -0.11 \\
$\%$ nREM & -0.26 & -0.09 & -0.23 & 0.04 & $-0.50^{* *}$ & -0.24 & -0.07 \\
Íar & -0.00 & 0.18 & 0.07 & 0.17 & -0.11 & 0.06 & -0.01 \\
IMC & $-0.39^{* *}$ & $-0.33^{* *}$ & $-0.46^{* *}$ & $-0.44^{* *}$ & $-0.38^{* *}$ & $-0.49^{* *}$ & 0.17 \\
\hline
\end{tabular}

Nota. IAH: índice de apnea-hipopnea; ID3: número de desaturaciones $>$ al 3\%; TST: tiempo total de sueño; \% REM: sueño REM/TST \%. \% no REM: sueño no REM/TST \%. Iar: índice de arousal; ESE: Escala de Somnolencia de Epworth. * $p<0.05$. Fuente: elaboración propia.

mente diferencias estadísticamente significativas entre ambos grupos $(t=8.30 ; p<0.01)$, situándose la puntuación media para el grupo clínico por encima del punto de corte de 10, mientras que la del grupo control queda muy por debajo del nivel de anormalidad, tomando como punto de corte la puntuación de 10.

\section{Relación de las variables}

polisomnográficas, respiratorias y el

IMC con el impacto funcional del sueño,

la somnolencia y la calidad de vida

En la Tabla 3 pueden observarse las correlaciones entre las puntuaciones del FOSQ, los niveles de somnolencia (ESE) y las variables respiratorias, polisomonográficas, así como el IMC. Con respecto a la somnolencia se encontró una correlación significativa entre esta última y el IAH $(r=0.29, p<0.05)$, así como con el número de desaturaciones superiores al 3\% (ID3) $(r=-0.35$, $p<0.05)$. En cuanto al impacto funcional del sueño, tal y como se muestra en los resultados, se encontraron correlaciones significativas entre el IMC (valores de $r$ entre $-0.31, p<0.01$ y -0.53 , $p<0.01)$ y todas las dimensiones medidas por este cuestionario, entre la ID3 y la productividad general $(r=-0.3 ; p<0.05)$, el nivel de actividad $(r=-0.29 ; p<0.05)$, la puntuación de sumario total $(r=-0.33 ; p<0.05)$, y entre el porcentaje de sueño REM y la dimensión relativa a la intimidad $(r=0.44 ; p<0.05)$.

En el análisis de regresión lineal por pasos, para los niveles de somnolencia, se incluyeron las variables de IAH e ID3. Resultó tener un efecto estadísticamente significativo $(t=2.541 ; p<0.05)$ solamente el ID3, con un coeficiente de orden cero de 0.347. También para el impacto funcional de sueño, el análisis de regresión lineal que se efectuó, incluyendo el ID3 y el IMC, resultó tener un efecto estadísticamente significativo solamente el IMC $(t=-3.498 ; p<0.01)$, con un coeficiente de orden cero de -0.451 . Los datos relativos a las variables de calidad de vida, su relación con las variables respiratorias y de sueño y el IMC, se muestran en la Tabla 4. Entre las variables respiratorias, resultaron significativas las correlaciones entre $\mathrm{la}_{\mathrm{SaO}}$ basal y la salud general, el rol emocional y sumatoria de salud mental (valores de $r$ entre $0.29, p<0.05$ y $0.33, p<0.05)$, mientras que el ID3 resultó relacionado con las variables de rol físico y de salud mental (valores de $r$ ente $-0.33 p<0.05$ y $0.35 p<0.05$ ). En cuanto a las variables de calidad de sueño, fue significativa la correlación entre los parámetros que valoran la cantidad del sueño (TST y eficiencia), la dimensión de salud mental $(r=0.29 p<0.05)$ 


\section{TABLA 4}

Correlaciones entre la calidad de vida (SF-36) y las variables polisomnográficas, respiratorias e indice de masa corporal (IMC) en el grupo de pacientes con SAHS

\begin{tabular}{lcccccccccc}
\hline \multicolumn{10}{c}{ Cuestionario de Salud SF-36 } \\
\hline & $\begin{array}{c}\text { Función } \\
\text { física }\end{array}$ & $\begin{array}{c}\text { Rol } \\
\text { físico }\end{array}$ & Dolor & $\begin{array}{c}\text { Salud } \\
\text { general }\end{array}$ & Vitalidad & $\begin{array}{c}\text { Función } \\
\text { Social }\end{array}$ & $\begin{array}{c}\text { Rol } \\
\text { Emocional }\end{array}$ & $\begin{array}{c}\text { Salud } \\
\text { Mental }\end{array}$ & $\begin{array}{c}\text { Sumatorio } \\
\text { Salud } \\
\text { física }\end{array}$ & $\begin{array}{c}\text { Sumatorio } \\
\text { Salud } \\
\text { mental }\end{array}$ \\
\hline $\mathrm{IAH}$ & -0.18 & -0.25 & -0.03 & -0.14 & -0.14 & -0.14 & -0.15 & -0.26 & -0.10 & -0.12 \\
$\mathrm{SaO}_{2}$ & 0.08 & 0.27 & 0.14 & $0.29^{*}$ & 0.14 & 0.24 & $0.33^{*}$ & 0.25 & 0.15 & $0.29^{*}$ \\
$\mathrm{ID} 3$ & -0.25 & $-0.35^{*}$ & -0.10 & -0.24 & -0.23 & -0.22 & -0.22 & $-0.33^{*}$ & -0.19 & -0.18 \\
$\mathrm{TST}$ & 0.25 & 0.20 & 0.20 & 0.25 & 0.21 & 0.21 & 0.23 & $0.29 *$ & 0.23 & 0.22 \\
$\mathrm{EF}$ & 0.24 & 0.23 & 0.16 & 0.23 & 0.24 & $0.29 *$ & 0.26 & $0.31^{*}$ & 0.26 & $0.32^{*}$ \\
$\% \mathrm{REM}$ & 0.21 & 0.25 & -0.00 & 0.24 & $0.40^{* *}$ & 0.25 & 0.23 & $0.36^{*}$ & 0.13 & $0.33^{*}$ \\
$\% \mathrm{REM}$ & -0.17 & -0.24 & 0.04 & -0.25 & $-0.42^{* *}$ & $-0.30^{*}$ & -0.27 & $-0.36^{*}$ & -0.09 & $-0.37^{* *}$ \\
$\mathrm{Iar}$ & 0.07 & 0.03 & 0.17 & 0.04 & 0.07 & 0.11 & -0.04 & -0.00 & 0.20 & 0.01 \\
$\mathrm{IMC}$ & $-0.47^{* *}$ & $-0.48^{* *}$ & $-0.38^{* *}$ & $-0.40^{* *}$ & $-0.43^{* *}$ & $-0.42^{* *}$ & $-0.50^{* *}$ & $-0.48^{* *}$ & $-0.40^{* *}$ & $-0.37^{* *}$ \\
\hline
\end{tabular}

Nota. IAH: índice de apnea-hipopnea; $\mathrm{SaO}_{2}$ : Saturación de oxígeno basal; ID3: número de desaturaciones superiores al 3 \%; TST: tiempo total de sueño; EF: eficiencia \% REM: sueño REM/TST \%; \% no REM: sueño no REM/TST \%. Iar: índice de arousal. $* p<0.05$.

Fuente: elaboración propia.

y el sumario de salud mental $(r=0.32 p<0.05)$, mientras que los parámetros que evalúan el porcentaje de sueño REM y no REM se correlacionaron con distintas dimensiones relativas a la vitalidad, a la salud mental, al sumario de salud mental y función social (valores de $r$ entre $-0.42 p<0.01$ y $0.40 p<0.01)$. El IMC correlacionó negativamente con todas las variables relativas a calidad de vida (valores de $r$ entre 0.37 y $0.5 ; p<0.01$ ).

\section{Discusión}

En esta investigación, realizada en un grupo de pacientes con SAHS y un grupo de sujetos sanos sin diagnóstico de SAHS, los resultados demuestran que el IMC, seguido de la gravedad de la hipoxemia nocturna (ID3), son las variables que más se relacionan con el deterioro en la calidad de vida y con el impacto que la somnolencia tiene en las actividades diarias de estos enfermos. En este trabajo se evidenció también una relación entre los niveles de somnolencia diurna y algunas variables de gravedad del síndrome como el IAH y el ID3.

Por otra parte, esta investigación muestra que los pacientes con SAHS informan de una peor calidad de vida comparada con los datos de referencia obtenidos en la población general española (Alonso et al., 1998), y que las puntuaciones del grupo con un SAHS son inferiores a las del grupo control en todas las subescalas, aunque las más afectadas son las de función física, salud general, vitalidad, índice general de salud física, salud mental y rol físico. Un estudio previo evidenció que los pacientes afectados por un SAHS obtuvieron menor puntuación en todas las subescalas del SF-36 que los sujetos de la población general (Mar et al., 2005). Similares resultados fueron aportados por otros autores que utilizaron el SF-36 (Moore, Bardwell, Ancoli-Israel \& Dimsdale, 2001; Sforza et al., 2003). Como se ha comentando previamente, estos resultados podrían indicar las importantes consecuencias del SAHS, a través de la hipoxemia intermitente (ID3), y la pobre calidad de sueño sobre los niveles de somnolencia diurna y del cansancio diurno experimentado por los pacientes con SAHS.

En la mayoría de los pacientes con un SAHS, se ha descrito excesiva somnolencia diurna (Seneviratne \& Puvanendran, 2004), aunque hay trabajos que la identifican solamente en un bajo porcentaje de sujetos con SAHS (Barbé et al., 2001; Kapur, Baldwin, Resnick, Gottlieb \& Nieto, 2005). Son pocos los estudios en la literatura que se hayan intere- 
sado por conocer la caracterización clínica de estos pacientes sin excesiva somnolencia. En este sentido, Campos-Rodríguez et al. (2010) encontraron que los pacientes con apnea sin excesiva somnolencia, muestran, en general, menor sintomatología diurna derivada de su apnea del sueño respecto al grupo de pacientes con somnolencia diurna; en concreto, los pacientes se quejaban menos de su deterioro intelectual y sueño poco reparador, a pesar de tener una gravedad similar en el SAHS.

En este estudio, el $74 \%$ de la población clínica informaba de niveles elevados de somnolencia. Los resultados además indican que los pacientes con SAHS presentan puntuaciones significativamente más elevadas en la ESE comparados con el grupo control. Los mismos resultados obtienen Sánchez y Buela-Casal (2007), al comparar la somnolencia de una muestra de pacientes con SAHS con una muestra de roncadores crónicos.

En relación con el impacto que la somnolencia tiene en las actividades diarias del paciente, se observa como las puntuaciones medias en el impacto funcional del sueño en el grupo clínico se encuentran muy por debajo de las del grupo control en todas las dimensiones medidas, aunque las mayores diferencias se obtienen en las de productividad general, nivel de actividad, vigilancia e intimidad. Estos datos están en la línea de los aportados por otros grupos (Büttner, Feier, Galetke \& Rühle, 2008; Vidal et al., 2007), lo que pone de manifiesto la importancia que la somnolencia diurna tiene en muchos aspectos de la vida diaria de los pacientes, principalmente en el trabajo, las relaciones sociales y la vida en pareja.

Por otra parte, se observa un evidente empeoramiento en la calidad de vida de los pacientes con SAHS. Sin embargo, los factores responsables son aún desconocidos, si bien se ha involucrado, entre otros aspectos, a la desestructuración del sueño, la somnolencia diurna, la depresión, la ansiedad y la apatía (Andrews \& Oei, 2004; Sforza et al., 2003). Moore et al. (2001) encontraron correlaciones significativas entre el tiempo total de sueño y seis dimensiones del Cuestionario de Salud MOS. Sin embargo, el IAH, utilizado habitualmente para la clasificación diagnóstica y de la gravedad del proce- so, no influyó en la calidad de vida de los enfermos. No obstante, es interesante destacar que variables relativas a la calidad de sueño como el porcentaje de sueño REM y no REM y la eficiencia, la saturación basal del oxigeno en la sangre, así como el IMC, muestran un importante impacto en la calidad de vida de estos pacientes.

En cuanto a la relación entre somnolencia y variables polisomnograficas, Roure et al. (2008) consideraron que las apneas y la fragmentación del sueño no son los factores más determinantes de este síntoma, y concluyeron que es la hipoxemia nocturna la que juega el papel más importante en la aparición de la somnolencia diurna. En nuestro estudio la variable relacionada con la somnolencia diurna se correlacionó, aunque de manera débil, con el IAH y el ID3. Un estudio reciente de Jiménez-Correa, Haro, González-Robles y Velázquez-Moctezuma (2010) pone en evidencia la fuerte relación entre gravedad del trastorno respiratorio del sueño y somnolencia. Por el contrario, Sierra, Sánchez, Buela-Casal, Ambrosio y Virués-Ortega (2006) no encontraron una correlación significativa entre el IAH y las puntuaciones en la ESE, concluyendo que probablemente el mejor predictor de la somnolencia no sea el número de apneas sino la duración de las mismas. Ishman, Cavey, Mettel y Gourin (2010) tampoco obtuvieron resultados que confirmaron una relación entre la somnolencia y el IAH.

El IMC es uno de los factores que más se asocian con el deterioro en la calidad de vida y en la funcionalidad diaria en pacientes diagnosticados de SAHS (Manrique et al., 2009). También Sforza et al. (2003) demostraron que, además de algunas variables de calidad de sueño, el IMC tiene una gran influencia en la alteración de la calidad de vida de los pacientes con SAHS.

La principal limitación de este estudio es que el cuestionario FOSQ, a pesar de estar validado en España con población española, no ha sido muy utilizado, por lo que son escasos los trabajos a los que se ha podido acceder para comparar los resultados obtenidos. No obstante, el número de muestra utilizado en ambos grupos permite aportar importantes hallazgos con relevancia clínica, entre los que destacan que, respecto al grupo control, los pacientes 
con SAHS revelan un significativo deterioro en la calidad de vida y en los niveles de somnolencia diurna. Las variables que presentan mayor correlación con las actividades diarias de los enfermos son los episodios bruscos de hipoxemia (valorada por el ID3) y el IMC. Estos resultados subrayan la importancia de estas variables sobre la salud de los pacientes, destacando el IMC que jugaría un papel central para convertir el SAHS en una enfermedad tan invalidante.

\section{Referencias}

Alonso, J., Prieto, L. \& Antó, J. M. (1995). La versión española del SF-36 Health Survey (Cuestionario de Salud SF-36): un instrumento para la medida de los resultados clínicos. Medicina Clínica, 104(20), 771-776.

Alonso, J., Regidor, E., Barrio, G., Prieto, L., Rodríguez, C. \& de la Fuente, L. (1998). Valores poblacionales de referencia de la versión española del Cuestionario de Salud SF-36. Medicina Clínica, 111(11), 410-416.

Andrews, J. G. \& Oei, T. P. (2004). The roles of depression and anxiety in the understanding and treatment of obstructive sleep apnea syndrome. Clinical Psychology Review, 24(8), 1031-1049.

Barbé, F., Mayorales, L. R., Duran, J., Masa, J. F., Maimó, A., Montserrat, J. M., et al. (2001). Treatment with continuous positive airway pressure is not effective in patients with sleep apnea but not daytime sleepiness. Annals of Internal Medicine, 134(11), 1015-1023.

Büttner, A., Feier, C., Galetke, W. \& Rühle, K. A. (2008). A questionnaire to capture the functional effects of daytime drowsiness on quality of life in case of obstructive sleep apnea syndrome. Functional Outcomes of Sleep Questionnaire (FOSQ). Pneumologie, 62(9), 548-552.

Campos-Rodríguez, F., Reina-González, A., ReyesNúñez, N., Beiztegui-Sillero, A., Almeida-González, C. \& Peña-Griñán, N. (2010). Características cardiovasculares y clínicas de los pacientes con apneas obstructivas del sueño sin somnolencia diurna excesiva. Archivos de Bronconeumología, 46(11), 594-599.
Chiner, E., Arriero, J., Signes-Costa, J., Marco, J. \& Fuentes, I. (1999). Validación de la versión española del Test de Somnolencia Epworth en pacientes con síndrome de apnea de sueño. Archivos de Bronconeumologia, 35(9), 422-427.

Ferrer, M., Vilagut, G., Monasterio, C., Montserrat, J. M., Mayos, M. \& Alonso, J. (1999). Medida del impacto de los trastornos del sueño: las versiones españolas del Cuestionario del Impacto Funcional del Sueño y de la Escala de Somnolencia de Epworth. Medicina Clínica, 113(7), 250-255.

Grupo Español de Sueño. (2005). Consenso nacional sobre el Síndrome Apnea-Hipopnea del Sueño (SAHS). Archivos de Bronconeumología, 41(Supl. 4), $12-29$.

Guglielmi, O., Sánchez, A. I., Jurado-Gámez, B., BuelaCasal, G. \& Bardwell, W. A. (2011). Obesidad y calidad de sueño: predictores de la depresión y la ansiedad en pacientes con Síndrome de ApneaHipopnea del Sueño. Revista de Neurología, 52(9), 515-521.

Ishman, S. L., Cavey, R. M., Mettel, T. L. \& Gourin, C. G. (2010). Depression, sleepiness, and disease severity in patients with obstructive sleep apnea. Laryngoscope, 120(11), 2331-2335.

Jiménez-Correa, U., Haro, R., González-Robles, R. O. \& Velázquez-Moctezuma, J. (2010). How is the Epworth Sleepiness Scale related with subjective sleep quality and polysomnographic features in patients with sleep-disordered breathing? Sleep Breathing, 15(3), 513-518.

Johns, M. W. (1991). A new method for measuring daytime sleepiness: The Epworth Sleepiness Scale. Sleep, 14(6), 540-545.

Kapur, V. K., Baldwin, C. M., Resnick, H. E., Gottlieb, D. J. \& Nieto, J. (2005). Sleepiness in patients with moderate to severe sleep-disordered breathing. Sleep, 28(4), 472-477.

Lópes, C., Esteves, A. M., Bittencourt, L. R., Tufik, S. \& Mello, M. T. (2008). Relationship between the quality of life and the severity of obstructive sleep apnea syndrome. Brazilian Journal of Medical and Biological Research, 41(10), 908-913.

Lurie, A. (2011). Obstructive sleep apnea in adults: Epidemiology, clinical presentation, and treatment options. Advances in Cardiology, 46, 1-42. 
Manrique, M., de la Maza, M. P., Carrasco, F., Moreno, M., Albala, C., García, J., et al. (2009). Diagnóstico, evaluación y tratamiento no farmacológico del paciente con sobrepeso u obesidad. Revista Médica de Chile, 137(7), 963-971.

Mar, J., Rivero-Arias, O., Durán-Cantolla, J., AlonsoÁlvarez, M. L., Gominde, I. \& de la Torre-Muñeca, G. (2005). Efecto del tratamiento con presión positiva continua en la vía respiratoria durante la noche en la calidad de vida de los pacientes con apnea del sueño. Medicina Clínica, 125(16), 611-615.

Marín-Agudelo, H. F., Franco, A. F., Vinaccia-Alpi, S., Tobón, S. \& Sandín, B. (2008). Trastornos del sueño, salud y calidad de vida: una perspectiva desde la medicina comportamental del sueño. Suma Psicologica, 15(1), 217-239.

McArdle, N. \& Douglas, N. J. (2001). Effect of continuous positive airway pressure on sleep architecture in the Sleep Apnea-Hypopnea Syndrome: A randomized controlled trial. American Journal of Respiration and Critical Care Medicine, 164(8), 1459-1463.

McArdle, N., Hillman, D., Beilin, L. \& Watts, G. (2007). Metabolic risk factors for vascular diseases in obstructive sleep apnea: A matched controlled study. American Journal of Respiratory and Critical Care Medicine, 175(2), 190-195.

Mediano, O., Barceló, A., de la Peña, M., Gozal, D., Agustí, A. \& Barbé, F. (2007). Daytime sleepiness and polysomnographic variables in sleep apnea patients. European Respiratory Journal, 30(1), 110-113.

Miró, E., Cano-Lozano, M. C. \& Buela-Casal, B. (2005). Sueño y calidad de vida. Revista Colombiana de Psicología, 14, 11-27.

Montero, I. \& León, O. G. (2007). A guide for naming research studies in Psychology. International Journal of Clinical and Health Psychology, 7(3), 847-62.

Moore, P., Bardwell, W. A., Ancoli-Israel, S. \& Dimsdale, J. E. (2001). Association between polysomnographic sleep measures and health-related quality of life in obstructive sleep apnea. Journal of Sleep Research, 10(4), 303-308.

Páez, S. \& Londoño, N. (2011). Síndrome de Apnea Hipoapnea obstructiva del sueño desde el punto de vista neumológico. Acta de Otorrinolaringología Eु Cirugía de Cabeza y Cuello, 39(3), 27-35.
Ramos-Álvarez, M. M., Moreno-Fernández, M. M., Valdés-Conroy, B. \& Catena, A. (2008). Criteria of the peer review process for publication of experimental and quasi-experimental research in psychology: A guide for creating research papers. International Journal of Clinical and Health Psychology, 8(3), 751-764.

Rechtschaffen, A. \& Kales A. (1968). A manual of standardized terminology, techniques, and scoring system for sleep stages of human subjects (NIH Publication No. 204). Washington, DC: Government Print Office.

Roure, N., Gómez, S., Mediano, O., Duran, J., de la Peña, M., Capote, F., et al. (2008). Daytime sleepiness and polysomnography in obstructive sleep apnea patients. Sleep Medicine, 9(7), 727-731.

Saldías, F. P., Jorquera, J. A. \& Díaz, O. P. (2010). Valor predictivo de la historia clínica y oximetría nocturna en la pesquisa de pacientes con apnea obstructiva del sueño. Revista Médica de Chile, 138(8), 941-950.

Sánchez, A. I. \& Buela-Casal, G. (2007). Assessment of daytime symptoms in snoring subjects and obstructive sleep apnea patients. Salud Mental, 30(1), 9-15.

Sánchez, A. I., Martínez, P., Miró, E., Bardwell, W. A. \& Buela-Casal, G. (2009). CPAP and behavioral therapies in patients with obstructive sleep apnea: Effects on daytime sleepiness, mood, and cognitive function. Sleep Medicine Reviews, 13(3), 223-233.

Sánchez, A. I., Miró, E. \& Martínez, P. (2006). Sindrome de apnea obstructiva del sueño y accidentes de tráfico. Revista Ecuatoriana de Neurología, 15(2-3), 86-94.

Seneviratne, U. \& Puvanendran, K. (2004). Excessive daytime sleepiness in obstructive sleep apnoea: Prevalence, severity, and predictors. Sleep Medicine, 5(4), 339-343.

Sforza, E., Janssens, J. P., Rochat, T. \& Ibanez, V. (2003). Determinants of altered quality of life in patients with sleep-related breathing disorders. European Respiratory Journal, 21(4), 682-687.

Sierra, J. C., Sánchez, A. I., Buela-Casal, G., Ambrosio, I. \& Virués-Ortega, J. (2006). Predictores del nivel de somnolencia en pacientes con apnea obstructiva del sueño. Revista Ecuatoriana de Neurología, 15(2-3), 80-85. 
Vidal, S., Ferrer, M., Masuet, C., Somoza, M., MartínezBallarín, J. I. \& Monasterio, C. (2007). Valores de la versión española del Cuestionario del Impacto Funcional del Sueño (FOSQ) en sujetos sanos y en pacientes con apnea obstructiva del sueño. Archivos de Bronconeumología, 43(5), 256-261.

Ware, J. E. \& Sherbourne, C. D. (1992). The MOS 36-item short-form health survey (SF-36). I. Con- ceptual framework and item selection. Medical Care, 30(6), 473-483.

Weaver, T., Laizner, A., Evans, L., Maislin, G., Chugh, D., Lyon, K., et al. (1997). An instrument to measure functional status outcomes for disorders of excessive sleepiness. Sleep, 20(10), 835-843. 
